\section{Comentario sobre los efectos de la estimulación cognitiva en la prevención y tratamiento de la demencia}

Commentary on the effects of cognitive
stimulation in the prevention and
treatment of dementia

Señor Editor:

La demencia es un problema de salud prioritario de abordar debido al aumento estimado de su prevalencia y a su impacto como principal causa de dependencia en población de 65 y más años. En dicho escenario, la evaluación de potenciales estrategias para su prevención, como también para su tratamiento en la trayectoria de progresión del deterioro cognitivo, son temas de alto interés científico y clínico. Entre estas, la estimulación cognitiva es una modalidad de intervención considerada con alta frecuencia. La Terapia de Estimulación Cognitiva (TEC) ${ }^{1}$ es una intervención estandarizada consistente en sesiones de involucramiento en actividades cognitivas y reminiscencia, estimulación multisensorial, y contacto social grupal, guiadas por un facilitador (no necesariamente profesional) con un modelo estándar de 14 sesiones grupales con frecuencia de 2 sesiones por semana durante 45 minutos por sesión.

En el documento titulado "Atención Integrada para Personas Mayores: Directrices para las intervenciones comunitarias dirigidas a limitar el deterioro de la capacidad intrínseca"2 la Organización Mundial de la Salud (OMS) plantea que "la terapia de estimulación cognitiva, como la participación en un rango de actividades con el fin de mejorar el funcionamiento social y cognitivo, es una estrategia crítica para prevenir y revertir el declive de la capacidad cognitiva, y consecuentemente, prevenir discapacidades funcionales y dependencia en la vejez" (p.14). En una primera lectura, dicho planteamiento puede parecer pertinente como sustento y aliciente para la implementación de esta estrategia. No obstante, al profundizar en el contenido científico del documento, se señala que la evidencia disponible para sustentar dicha recomendación es baja definiendo su uso condicional, lo que quiere decir que no existen suficientes investigaciones de alta calidad que informen sus resultados, que existen estimaciones imprecisas de los beneficios y riesgos, y/o que los beneficios son muy acotados y reducidos en relación con su costo. Con dichos antecedentes, resulta llamativo que se afirme el potencial preventivo, e incluso recuperativo, de la TEC en el deterioro cognitivo adquirido, en un escenario global de limitado avance en la búsqueda de tratamientos, incluso farmacológicos, que demuestren dicho efecto considerando la base neuropatológica de este problema de salud. ¿Por qué se atribuye este potencial no obstante la baja evidencia científica referida? ¿Por qué usualmente se considera esa estrategia como primera línea de prevención, por sobre otras? ¿Corresponde su implementación a una práctica con base en evidencia, o una práctica basada en la costumbre?

Al respecto, se dispone de relevantes documentos que han buscado sintetizar evidencia científica sobre el potencial alcance preventivo y recuperativo de diversas estrategias no farmacológicas en deterioro cognitivo, incluyendo la TEC, en momentos clave de la trayectoria de deterioro cognitivo: conversión perfil sano a deterioro cognitivo leve, conversión deterioro cognitivo leve a demencia, y finalmente, progresión del deterioro en demencia ${ }^{3-5}$. De manera consistente, la evidencia científica disponible no permite afirmar que la TEC, o cualquier forma de estimulación cognitiva no estandarizada, por sí sola o como estrategia central de intervenciones multicomponente, logra prevenir o revertir el deterioro cognitivo leve, así como tampoco el trastorno neurocognitivo mayor. Gran parte de los ensayos clínicos de TEC presentan limitaciones en la interpretación de sus resultados, asumiendo que la mejoría de un dominio específico de la cognición implica transferencia y mejoría en el rendimiento global, o que la mejoría evaluada por pautas (como MMSE) se correlaciona con un efecto clínico. Importantes ensayos clínicos han reportado, además, pérdida de efecto a los 2 años y nulos efectos en la memoria ${ }^{6}$.

De igual modo, los programas individuales de entrenamiento cerebral o cognitivo (que buscan el 
mejoramiento unidimensional inmediato luego de entrenamiento), atractivos por su frecuente modalidad de software o material de estimulación, han sido escasamente evaluados y no han demostrado su eficacia ${ }^{5}$. Tanto en TEC como en entrenamiento cerebral o cognitivo se resalta, además, la insuficiente claridad en sus bases, contrastando con las teorías más aceptadas sobre el deterioro cognitivo (como la reducción de factores neuropatogénicos, la neuroplasticidad, la neurogénesis, y los biomarcadores). Al respecto, la naturaleza grupal de la intervención levanta una pregunta esencial en la evaluación de las vías de efecto y causalidad de sus potenciales efectos. ¿Podría pensarse que es el efecto grupo el principal modulador de sus resultados? Esto es más aún pertinente al constar que ensayos clínicos de TEC han probado ser eficaces en la mejoría de calidad de vida y otras variables de bienestar psicosocial ${ }^{1,3}$. Según las diferentes dimensiones de intervención que implica la TEC, es necesario observar sus potenciales mediadores, sobresaliendo entre estos la potencial influencia relativa de la activación conductual ${ }^{7,8}$. Lo anterior, levanta oportunidades de investigación hacia la redefinición de sus bases conceptuales y teorías que expliquen vías de efecto y resultados.

Por lo anterior, debe potenciarse la transferencia del conocimiento actualmente disponible sobre prevención de las demencias, que enfatiza en estrategias bioconductuales para la reducción de factores de riesgo modificables de salud cardiovascular y metabólica, y también de dimensiones sociales y afectivas. Dichos factores debiesen abordarse no solo en la adultez mayor, sino con gran relevancia en la adultez media, relevando la importancia de incorporar el curso de vida como un determinante clave en la prevención de los trastornos cognitivos.

Luego, por sobre focalizar esfuerzos en la implementación de esta modalidad específica de intervención, debiese potenciarse una mirada amplia de los factores de riesgo modificables para demencia en la adultez media y mayor, entre los que destacan el acceso a tratamiento para la depresión, la compensación de déficit auditivo, el manejo de diabetes mellitus e hipertensión arterial, la reducción del aislamiento social, la realización de ejercicio físico y la reducción del consumo de tabaco (como moduladores de riesgo neuropatogénico cardiovascular y metabólico $)^{3}$, fortaleciendo la acción integrada de los diferentes programas preventivos existentes, tales como el Programa Elige Vivir Sano y el Programa Más Adultos Mayores Autovalentes.

Por su parte, en relación con el efecto de TEC y otras intervenciones no farmacológicas en demencias ya establecidas, la evidencia científica disponible sugiere que su principal efecto se relaciona con modular el impacto psicosocial del problema de salud (riesgos de accidentes, calidad de vida, efectos negativos en cuidador, síntomas neuropsiquiátricos, uso de recursos en la comunidad, entre otros). De este modo, al igual que la terapia farmacológica disponible, la evidencia científica existente no permite afirmar que las terapias no farmacológicas posean un efecto modificador de la enfermedad en la cognición y la capacidad funcional, principales indicadores clínico de su progresión o potencial recuperación?.

La definición de políticas públicas y programas, al igual que los(as) profesionales y equipos vinculados a la prevención, promoción e intervención con personas con demencia, debemos ser cautelosos con las expectativas del impacto atribuible a las intervenciones, tanto farmacológicas como no farmacológicas, potencialmente existentes o instaladas como estrategias en el sistema de salud, promoviendo aquellas que disponen de mayor nivel de recomendación y que informan el efecto en la carga de enfermedad y calidad de vida, por sobre la modificación clínica de su progresión. Del mismo modo, debe continuar potenciándose la investigación que informe sobre los efectos y riesgos de la TEC y otras intervenciones no farmacológicas en demencias.

Jean Gajardo J. ${ }^{1}$

Facultad de Medicina, Universidad San Sebastián Departamento de Terapia Ocupacional y Ciencia de la Ocupación, Facultad de Medicina,Universidad de Chile.

${ }^{1}$ Terapeuta Ocupacional. Doctor en Salud Pública. E-mail: jean.gajardo@gmail.com 


\section{Referencias bibliográficas}

1. Aguirre E, Woods RT, Spector A, Orrell M. Cognitive stimulation for dementia: a systematic review of the evidence of effectiveness from randomised controlled trials. Ageing Res Rev [Internet]. 2013 Ene [citado el 14 de agosto de 2014]; 12 (1): 25362. Disponible en: http://www.ncbi.nlm.nih.gov/ pubmed/22889599.

2. World Health Organization. Integrated care for older people: guidelines on community-level interventions to manage declines in intrinsic capacity [Internet]. Geneva; 2017. 46 p. Disponible en: http://www.who.int/ageing/publications/ guidelines-icope/en/

3. Livingston G, Sommerlad A, Orgeta V, Costafreda SG, Huntley J, Ames D, et al. Dementia prevention, intervention, and care [Internet]. The Lancet. 2017. Disponible en: http://www.thelancet.com/ commissions/dementia2017.

4. Butler M, McCreedy E, Nelson VA, Desai P, Ratner E, Fink HA, et al. Does cognitive training prevent cognitive decline? Ann Intern Med 2018; 168: 63-8.

5. Kane RL, Butler M, Fink HA, Brasure M, Davila $H$, Desai $P$, et al. Interventions To Prevent Age-Related Cognitive Decline, Mild Cognitive Impairment, and Clinical Alzheimer's-Type De- mentia [Internet]. 2017. Disponible en: https:// www.ncbi.nlm.nih.gov/books/NBK442425/pdf/ Bookshelf_NBK442425.pdf.

6. Tennstedt SL, Unverzagt FW. The ACTIVE Study: Study Overview and Major Findings. J Aging Health [Internet]. 2013; 25(8 Suppl):3S20S. Disponible en: http://jah.sagepub.com/cgi/ doi/10.1177/0898264313518133.

7. Gitlin LN, Roth DL, Huang J. Mediators of the Impact of a Home-based Intervention (Beat the Blues) on Depressive Symptoms among Older African Americans. Psychol Aging 2014; 29 (3): 601-11.

8. Kanter JW, Manos RC, Bowe WM, Baruch DE, Busch AM, Rusch LC. What is behavioral activation? A review of the empirical literature. Clin Psychol Rev [Internet]. 2010; 30 (6): 60820. Disponible en: http://dx.doi.org/10.1016/j. cpr.2010.04.001.

9. Webster L, Groskreutz D, Grinbergs-Saull A, Howard R, O’Brien JT, Mountain G, et al. Core outcome measures for interventions to prevent or slow the progress of dementia for people living with mild to moderate dementia: Systematic review and consensus recommendations. PLoS One [Internet] 2017; 12 (6): e0179521. Disponible en: http://dx.doi.org/10.1371/journal.pone.0179521. 